Modern Physics Letters A

(C) World Scientific Publishing Company

\title{
THERMALLY EXCITED QUASIPARTICLES IN METALS, DISPERSION FORCES, AND THE THERMAL ANOMALY
}

\author{
CARSTEN HENKEL \\ Institute of Physics and Astronomy, University of Potsdam, \\ Karl-Liebknecht-Str. 24/25, 14476 Potsdam, Germany \\ henkel@uni-potsdam.de
}

\begin{abstract}
We provide a brief review of the contribution of thermally excited carriers to dispersion forces. In a metal, these carriers generate charge and current fluctuations whose spectral frequencies are comparable to $k_{B} T / \hbar$. They are very likely responsible for the "plasma vs. Drude" anomaly.

Keywords: Dispersion force; quasi-particles.

PACS Nos.: 42.50.Pq, 42.50.Lc, 44.40.+a
\end{abstract}

\section{Dispersion Forces at Different Distances and Relevant Frequencies}

There is a long history behind the temperature dependence of dispersion forces. The physical problem is easy to formulate: since the pioneering work of Casimir ${ }^{1}$ who switched from the viewpoint of van der Waals forces, generated by dipole fluctuations of material particles, to the viewpoint of field modes and their zeropoint energy, it is natural to ask how thermally excited states of matter or of field modes participate in the momentum exchange between two objects.

For a given physical system it is relatively easy to decide whether thermal occupation plays a role or not: within the frequency spectrum of the relevant modes, those with $\hbar \omega \gg k_{B} T$ are essentially in the quantum regime and contribute with their zero-point energies, while frequencies $\hbar \omega \ll k_{B} T$ are enhanced by a factor scaling with $k_{B} T / \hbar \omega$ at low frequencies. Since most experiments are performed at room temperature $T \sim 300 \mathrm{~K}$, one gets the characteristic far-infrared value $\omega_{T} / 2 \pi=k_{B} T /(2 \pi \hbar) \sim 26 \mathrm{meV}=6.2 \mathrm{THz}=208 \mathrm{~cm}^{-1}$.

The relevant frequency spectrum is not trivial to find because dispersion forces are naturally generated by a continuum of frequencies - remember the theory of Eisenschitz and London for the van der Waals force with its integration over virtual transitions reaching even into the ionisation continuum. ${ }^{2}$ In the Casimir effect between parallel plates, an electrodynamic scale emerges naturally, namely the cutoff frequency for a cavity of length $d, \omega_{d}=\pi c / d$. The distinction between "quantum" and "thermal" regimes thus maps into short and large distances (see Table 1 and Fig. 1), separated quite accurately by the scale $\lambda_{T}=c / 2 \omega_{T}$, giving at room temperature $\lambda_{T} \sim 3.8 \mu \mathrm{m}$. This sets a challenge for experiments with macroscopic bodies because dispersion forces are weak, and relatively accurate control over the geome- 
try (parallelism of plates) is required. The scaling of the Casimir free energy $\mathcal{F}$ per unit area between two large plates can be found by dimensional analysis in three regimes, as shown in Table 1.

Experiments at closer distances benefit from larger forces, and one expects them to be dominated by the zero-point fluctuations of matter and field. It thus came as a surprise that in 2000, Boström and Sernelius reported significant thermal corrections to the Casimir pressure between metallic plates at distances smaller than the thermal wavelength. ${ }^{3}$ At about the same time, Svetovoy and Lokhanin worked on precision calculations and also had to take into account both temperature and material parameters. ${ }^{4,5}$ This period saw the birth of the "thermal anomaly" in the Casimir effect. By the anomaly, we mean the unusually large thermal correction that already appears in the intermediate range $\lambda_{p} \lesssim d \ll \lambda_{T}$. After the experimental work by the groups of Lamoreaux and of Mohideen, additional experiments were reported, ${ }^{6-11}$ and a comparison to theory at the few-percent level seemed within reach. In earlier periods, theorists checked their calculations by considering the special case of a cavity with perfectly conducting boundaries. This case had been studied for example by Mehra ${ }^{12}$ and by Schwinger, DeRaad, and Milton ${ }^{13}$ as a limiting case. Schwinger and co-workers provided a critical survey of asymptotic expressions worked out by Lifshitz, ${ }^{14}$ in particular for the finite-temperature case:

[...] we have verified Lifshitz' formula ${ }^{14}$ for the Casimir force between parallel dielectric interfaces, including the temperature dependence. Where the Russian calculations went wrong ${ }^{15}$ was in their specialization to metal plates. A careful reading of their papers shows that, through relatively trivial errors, they obtained incorrect results for the Casimir force, for perfect conductors at finite temperatures, and for imperfect conductors at zero temperature.

In this paper, we review the physical origin of the unexpected temperature dependence of the dispersion force between imperfect conductors.

\section{Lifshitz Theory and Thermal Casimir Pressure Correction}

The theoretical work after Lifshitz confirmed the validity of his celebrated formula for the Casimir pressure $P$ between two plates. We copy it here for plates 1 and 2

Table 1. Distance regimes and Casimir free energy between two metallic plates. See text for definitions.

\begin{tabular}{ccc}
\hline quantum $=$ short distance: $d \ll \lambda_{T}$ & thermal $=$ large distance: $d \gg \lambda_{T}$ \\
\hline non-retarded: $d \ll \lambda_{p}$ & retarded: $\lambda_{p} \ll d$ & \\
\hline $\mathcal{F} \sim-\frac{\hbar \omega_{p}}{d^{2}}$ & $\mathcal{F} \sim-\frac{\hbar c}{d^{3}}$ & $\mathcal{F} \sim-\frac{k_{B} T}{d^{2}}$ \\
\hline
\end{tabular}


as an integral over real frequencies

$$
P(d, T)=\int_{0}^{\infty} \frac{\mathrm{d} \omega}{2 \pi}\left[\frac{1}{2}+\bar{n}(\omega, T)\right] \int_{\mathrm{L}} \frac{k_{z} \mathrm{~d} k_{z}}{2 \pi} \operatorname{Re}\left(2 \hbar k_{z} \sum_{\sigma=\mathrm{p}, \mathrm{s}} \frac{r_{1 \sigma} r_{2 \sigma} \mathrm{e}^{2 \mathrm{i} k_{z} d}}{1-r_{1 \sigma} r_{2 \sigma} \mathrm{e}^{2 \mathrm{i} k_{z} d}}\right),
$$

where $\bar{n}(\omega, T)$ is the Bose distribution, providing a natural cutoff $\omega \lesssim \omega_{T}$ for the thermal contribution; the $k_{z}$-integral starts at $k_{z}=\omega / c$ and goes via the origin to $k_{z}=+\mathrm{i} \infty$ (symbolically written $\mathrm{L}$ ). In particular the works by Kats and Bimonte made it clear that the $r_{\sigma}$ are indeed reflection amplitudes for electromagnetic waves in the $\mathrm{p}(\mathrm{TM})$ and $\mathrm{s}(\mathrm{TE})$ polarisation. ${ }^{16,17}$ The same expression applies for zero-point and thermal fluctuations, apart from the different frequency ranges that dominate the two contributions. Expanding in powers of $r_{1 \sigma} r_{2 \sigma}$, we get a multiple reflection sum, the basis for the scattering approach to the Casimir pressure. ${ }^{18}$ Perfect reflectors give $r_{\mathrm{s}}=-1$ and $r_{\mathrm{p}}=+1$, and the denominator constrains the integral to cavity modes with $k_{z}=n \pi / d$; imaginary $k_{z}$ (evanescent waves) then do not contribute.

For illustration, we plot in Fig. 1 the difference $P(d, T)-P(d, 0)$ of the Casimir pressure between perfectly conducting plates. ${ }^{12}$ The thick line is the exact result, the dot-dashed line gives for comparison the zero-temperature pressure $P(d, 0)$, the gray dashed line the high-temperature asymptote $P(d, T) \simeq-\zeta(3) k_{B} T /\left(4 \pi d^{3}\right)$ that dominates for $d \gg \lambda_{T}$, as expected. At smaller distances, it is, for perfect reflectors, a tiny correction, since at $d=1 \mu \mathrm{m}, P(d, 0)=-1.3 \mathrm{mPa}$.

Any realistic material brings in a characteristic cutoff beyond which it is transparent, as Casimir noted himself. The reflection coefficients encode these material properties, as they must, since electromagnetic vacuum fluctuations manifest themselves only in so far they couple to matter. ${ }^{19,20}$ For gold, the cutoff is set by the plasma frequency $\omega_{p} / 2 \pi \simeq 9 \mathrm{eV}$, as computed from the free carrier density. The plasma wavelength $\lambda_{p}=c / \omega_{p} \sim 22 \mathrm{~nm}$ (also known as Meissner penetration depth) is not a microscopic length compared to the closest distances $d \sim 100 \mathrm{~nm}$ achieved in recent Casimir force experiments. The regime $d \ll \lambda_{p}$ is quantum rather than thermal, non-retarded and amenable to the standard approximations of theoretical chemistry, even though van der Waals interactions need special tools like the adiabatic connection theorem to be reached with density functional techniques, for example. ${ }^{21,22}$

\section{Nature and Contribution of Matter-Dominated Modes}

What is the impact of the material parameters on the Casimir force? This question has been at the heart of the debate around its temperature dependence. It is related to the question what kind of quasi-particles may contribute to the force, in addition to the electromagnetic field. Looking at reflection coefficients, a non-ideal medium broadens the resonances that would be infinitely sharp between two perfect conductors, and makes them disappear above the plasma (or transparency) edge. Also new types of electromagnetic quasi-particles appear, like surface plasmon polaritons, lo- 


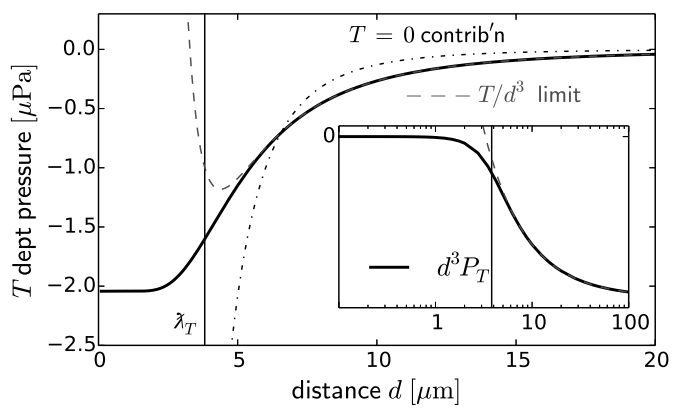

Fig. 1. Temperature-dependent Casimir pressure between two perfectly conducting plates. ${ }^{12}$ The gray dashed line is the difference between the high-temperature $T / d^{3}$ and the zero-temperature $\hbar c / d^{4}$ asymptotes. Inset: pressure multiplied by $d^{3}$. Negative values correspond to an attractive force.

cated at $\omega_{p} / \sqrt{2}$ (and below) at a metal-vacuum interface. ${ }^{23,24}$ Their hybridization provides a simple way to understand the Casimir pressure in terms of "bonding" and "anti-bonding modes", leading to the scaling $\mathcal{F} \sim \hbar \omega_{p} / d^{2}$ (Table 1). ${ }^{25-27}$ Surface polaritons being bound to the metal surface, they correspond to purely imaginary wave vectors $k_{z}$; this 'leg' of the d $k_{z}$-integral in Eq. (1) (evanescent waves in the TM polarization) thus becomes dominant at distances $d \sim \lambda_{p}$.

It has been argued that electric field fluctuations of very low frequencies inside a metal cannot be relevant for the Casimir force because of the fast response of the charge density on its boundaries. ${ }^{28}$ The estimate is based on Ohm's law $\mathbf{j}=\sigma \mathbf{E}$ that gives, combining with charge conservation and Coulomb's law

$$
\partial_{t} \rho=-\nabla \cdot \sigma \mathbf{E}=-\frac{\sigma}{\varepsilon_{0}} \rho .
$$

This would give a time scale $\varepsilon_{0} / \sigma \sim 10^{-19} \mathrm{~s}$ (for gold) after which the total electric field in plates of finite size has decayed to zero. One may raise the question whether for such a fast decay, the DC conductivity is still meaningful. Indeed, a different time scale is found from Drude's model for the current density

$$
\partial_{t} \mathbf{j}+\frac{\mathbf{j}}{\tau}=\frac{\sigma}{\tau} \mathbf{E}
$$

with a relaxation time $\tau$. Taking the divergence leads to the telegraphist's equation

$$
\partial_{t}^{2} \rho+\frac{\partial_{t} \rho}{\tau}+\frac{\sigma}{\varepsilon_{0} \tau} \rho=0
$$

that yields an eigenfrequency with imaginary part $-1 /(2 \tau)$ : the charge density cannot relax faster than the current density. These processes cannot be irrelevant because their rate is quite comparable to the room-temperature characteristic frequency $\omega_{T}$ : a typical value for gold is $\tau \sim 27 \mathrm{fs}$ so that $1 /(2 \pi \tau) \sim 24 \mathrm{meV}=$ $5.9 \mathrm{THz}=190 \mathrm{~cm}^{-1}$. (With respect to spatial scales, one may raise doubts about the relevance of the length scale $\varepsilon_{0} c / \sigma \sim 1 \AA$, that has led to the claim that the con- 
ductivity should be irrelevant for macroscopic distances involved in typical Casimir forces. ${ }^{5}$ )

The Drude conductivity that follows from Eq. (3)

$$
\sigma(\omega)=\frac{\sigma}{1-\mathrm{i} \omega \tau},
$$

has been discussed in many papers and conferences. ${ }^{29-38}$ It has been argued that for Casimir calculations, the lossless variant, the "plasma model"

$$
\sigma(\omega)=\frac{\mathrm{i} \sigma}{\omega \tau}=\frac{\mathrm{i} \varepsilon_{0} \omega_{p}^{2}}{\omega},
$$

should be used, where the Drude parameters combine into the plasma frequency, excluding actual Ohmic losses. This is not so obvious for the thermal correction that arises from $\omega \ll \omega_{T} \sim 1 / \tau$ where Eq. (6) is a poor approximation.

We are touching here those modes to which the thermal anomaly can be attributed: it was clear from the beginning that they are TE-polarized and appear at low frequencies. ${ }^{3,5,39-41}$ An inspection of Eq. (1) shows that they appear for imaginary $k_{z}$ (evanescent waves). In Ref. 42 , it was explicitly stated that these modes correspond to magnetic fields. This insight also provides a physical explanation why they are not efficiently reflected by a metallic surface: by the Bohr-von Leeuwen theorem, a metal is transparent to static magnetic fields, unless quantum effects like superconductivity play a role. ${ }^{43}$ The consequences of this behaviour can be seen directly in the large-distance behaviour of the Casimir free energy computed within classical field theory: ${ }^{44,45}$ the power law $\mathcal{F} \sim k_{B} T / d^{2}$ comes with a prefactor that is half as large compared to the result obtained with perfectly reflecting bodies. Also the plasma model (6) yields a non-zero static result for the reflection coefficient $r_{\mathrm{s}}$ when the conventional Fresnel formula is used:

$$
r_{\mathrm{s}}=\frac{k_{z}-k_{z m}}{k_{z}+k_{z m}}, \quad k_{z m}=\sqrt{\mathrm{i} \mu_{0} \omega \sigma(\omega)+k_{z}^{2}}=\sqrt{k_{z}^{2}-1 / \lambda_{p}^{2}} .
$$

This has led Intravaia and the present author to the statement that the imaginary conductivity of Eq. (6) actually describes a superconductor with its distinct feature of the Meissner effect. (It is obvious, of course, that this statement is only true at low frequencies. The conductivity of a BCS superconductor becomes complex at frequencies above the gap, for example.)

\section{Contribution of Diffusing Magnetic Fields}

If a normal conductor is penetrable to low-frequency magnetic fields, how is it possible that these fields contribute to the Casimir effect? They are evanescent and contribute to the Lifshitz formula along imaginary $k_{z}=\mathrm{i} \kappa$. Using the Drude conductivity in the Fresnel formula then yields instead of Eq. (7) $\left(\operatorname{Im} k_{z m} \geq 0\right)$

$$
r_{\mathrm{s}}=\frac{\mathrm{i} \kappa-k_{z m}}{\mathrm{i} \kappa+k_{z m}}, \quad k_{z m}=\sqrt{\frac{\mathrm{i} \omega / D}{1-\mathrm{i} \omega \tau}-\kappa^{2}}, \quad D=\frac{1}{\mu_{0} \sigma}=\frac{\lambda_{p}^{2}}{\tau} .
$$


Here, the quantity $D$ is the magnetic diffusivity that describes how time-dependent magnetic fields penetrate into a normal conductor (a standard non-invasive technique for material inspection called eddy current testing). This class of modes is illustrated in Fig. 2, where the real part of the last parenthesis in Eq. (1) is shown, evaluated for $k_{z}=\mathrm{i} \kappa$ and multiplied by $\kappa$. The vertical line gives the natural cutoff $\kappa=1 / d$. One notes that the peak (central contour) is located near $\omega \sim 1 / \tau$.

The thermal anomaly in the Casimir force thus arises from thermal current fluctuations that diffuse across a metallic body, partially cross its surface and couple in the form of evanescent magnetic fields to another metallic body. These modes are therefore not surface, but bulk "modes", and have a peculiar status because their eigenfrequencies are purely imaginary, $\omega_{k}=-\mathrm{i} D k^{2}$. For $k \lambda_{p} \ll 1$, this scaling law provides a natural upper limit for magnetic bulk fields, as shown in dashed in Fig. 2, projected onto real frequencies. In thermal equilibrium, the damping of fields applied from outside the body is exactly compensated by the fields generated from thermal fluctuating currents by Ampere's law. ${ }^{46,47}$ Ingold and co-workers have worked out in detail the free energy of an overdamped particle with an imaginary eigenfrequency. ${ }^{36,48,49}$ It also shows thermal anomalies like a negative specific heat or entropy. These arise from the subtraction of the heat bath coupled to the overdamped particle.

The quantum field theory of overdamped magnetic field modes has also been worked out. ${ }^{50-53}$ It has been shown that the relevant electromagnetic mode density in the two-plate cavity (easy to read off from Eq. (1)) is essentially exhausted in spectral weight for $\omega \lesssim 1 / \tau$ by diffusive magnetic fields. This has clarified the negative entropies of the Casimir problem: ${ }^{54,55}$ they correspond to a tiny, surfacedependent entropy shift of magnetic fields diffusing throughout the bulk. ${ }^{50,51}$

In discussions of the thermal anomaly, media with temperature-dependent scattering times have been put forward. For a perfect crystal, one expects $\tau(T) \rightarrow \infty$ as $T \rightarrow 0$ because all scattering mechanisms freeze out. The Casimir entropy has been worked out in this limit ${ }^{50,51}$ and found to be of the order of $S \sim-k_{B} / d^{2}$. This was claimed a violation of the Nernst heat theorem that tells us that the entropy of an isolated body should vanish at absolute zero. ${ }^{55}$ For this specific case, magnetic diffusion could resolve the paradox: ${ }^{51}$ when taking the limit, the frequency range $\omega \lesssim 1 / \tau(T)<\omega_{T}$ where these modes can be excited moves to ever lower frequency, but always stays in the thermal regime. This is an example of a subtle interplay of taking the zero-temperature or zero-frequency limits. ${ }^{56}$ The perfect crystal thus degenerates into a "glassy state" filled with current loops interlocked with magnetic field lines. This theoretical example of a perfect (rather than super-)conductor contains a surface-dependent disorder entropy because, as mentioned above, magnetic fields in the two bodies can couple to each other via the vacuum gap. 


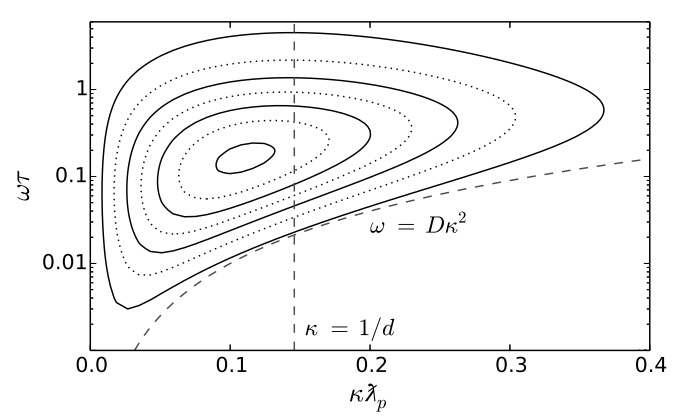

Fig. 2. Mode-resolved Casimir pressure between two Drude metals as a function of imaginary wave vector $k_{z}=\mathrm{i} \kappa$ and real frequency $\omega$ (see main text for details). Typical Drude parameters for gold $\left(\omega_{p} \tau \approx 390\right.$, magnetic diffusivity $\left.D \approx 0.018 \mathrm{~m}^{2} / \mathrm{s}\right)$ and distance $d=150 \mathrm{~nm}=6.9 \lambda_{p}$.

\section{Conclusion}

To conclude, the challenge remains to explain the discrepancy between Lifshitz theory for non-perfect conductors and micromechanical experiments in Decca's group $^{57-60}$ and atomic force microscope experiments in Mohideen's group. ${ }^{61-63}$ Theoretical limits like the perfect conductor or the Nernst theorem are of little help, since one has to deal with a real system here. We conjecture that surface roughness may yield surprises for the particular class of low-frequency magnetic near fields (in the $\mathrm{THz}$ band and on the $10-100 \mathrm{~nm}$ spatial scale) that is not yet directly amenable to spectroscopic experiments.

\section{Acknowledgments}

This work has been supported by the Deutsche Forschungsgemeinschaft through the DIP program (grant nos. Schm 1049/7-1 and Fo 703/2-1).

\section{References}

1. H. B. G. Casimir, Proc. Kon. Ned. Akad. Wet. 51, 793 (1948).

2. R. Eisenschitz and F. London, Z. Phys. 60, 491 (1930).

3. M. Boström and B. E. Sernelius, Phys. Rev. Lett. 84, 4757 (2000).

4. V. B. Svetovoy and M. V. Lokhanin, Mod. Phys. Lett. A 15, 1437 (2000).

5. V. Svetovoy and M. Lokhanin, Phys. Lett. A 280, 177 (2001).

6. S. K. Lamoreaux, Phys. Rev. Lett. 78, 5 (1997), erratum: 81 (1998) 5475.

7. U. Mohideen and A. Roy, Phys. Rev. Lett. 81, 4549 (1998).

8. B. W. Harris, F. Chen and U. Mohideen, Phys. Rev. A 62, 052109 (2000).

9. G. Bressi, G. Carugno, R. Onofrio and G. Ruoso, Phys. Rev. Lett. 88, 041804 (2002).

10. R. S. Decca, D. López, E. Fischbach and D. E. Krause, Phys. Rev. Lett. 91, 050402 (2003).

11. F. Chen, G. L. Klimchitskaya, U. Mohideen, and V. M. Mostepanenko, Phys. Rev. A 69, 022117 (2004).

12. J. Mehra, Physica 37, 145 (1967).

13. J. Schwinger, L. L. DeRaad, Jr. and K. A. Milton, Ann. Phys. (N.Y.) 115, 1 (1978).

14. E. M. Lifshitz, Soviet Phys. JETP 2, 73 (1956) [Zh. Eksp. Teor. Fiz. 29, 94 (1955)]. 
15. C. M. Hargreaves, Proc. Kon. Ned. Akad. Wetensch. B 68, 231 (1965)]

16. E. I. Kats, JETP Soviet Phys. 46, 109 (1977).

17. G. Bimonte and E. Santamato, Phys. Rev. A 76, 013810 (2007).

18. S. Reynaud, A. Canaguier-Durand, R. Messina, A. Lambrecht and P. A. Maia Neto, Int. J. Mod. Phys. A 25, 2201 (2010).

19. R. L. Jaffe, Phys. Rev. D 72, 021301(R) (2005).

20. H. Nikolić, Phys. Lett. B 761, 197 (2016).

21. R. A. DiStasio Jr, V. V. Gobre and A. Tkatchenko, J. Phys.: Condens. Matt. 26, $213202(2014)$.

22. L. M. Woods, D. A. R. Dalvit, A. Tkatchenko, P. Rodriguez-Lopez, A. W. Rodriguez and R. Podgornik, Rev. Mod. Phys. 88, 045003 (2016).

23. G. Barton, Rep. Progr. Phys. 42, 963 (1979).

24. H. Raether, Surface Plasmons on Smooth and Rough Surfaces and on Gratings, Springer Tracts in Modern Physics, Vol. 111 (Springer, 1988).

25. C. Henkel, K. Joulain, J.-P. Mulet and J.-J. Greffet, Phys. Rev. A 69, 023808 (2004).

26. A. I. Volokitin and B. N. J. Persson, Phys. Rev. B 69, 045417 (2004).

27. F. Intravaia and A. Lambrecht, Phys. Rev. Lett. 94, 110404 (2005).

28. B. Geyer, G. L. Klimchitskaya and V. M. Mostepanenko, J. Phys. A: Math. Theor. 40, 13485 (2007).

29. V. M. Mostepanenko, V. B. Bezerra, R. S. Decca, B. Geyer, E. Fischbach, G. L. Klimchitskaya, D. E. Krause, D. López and C. Romero, J. Phys. A: Math. Gen. 39, 6589 (2006).

30. M. Bordag and V. M. Mostepanenko (eds.), Papers Presented at the 8th Workshop QFExt07, J. Phys. A: Math. Theor. 41, No 16 (2008).

31. G. L. Klimchitskaya, U. Mohideen and V. M. Mostepanenko, Rev. Mod. Phys. 81, 1827 (2009).

32. G. L. Klimchitskaya, J. Phys.: Conf. Series 161, 012002 (2009).

33. K. Milton and M. Bordag (eds.), Selected Papers from the 9th Conference QFExt09, Int. J. Mod. Phys. A 25, No 11 (2010).

34. K. A. Milton, I. Brevik and S. Å. Ellingsen, Phys. Scr. 2012, 014070 (2012).

35. I. Brevik and J. S. Høye, Eur. J. Phys. 35, 015012 (2014).

36. K. A. Milton, Y. Li, P. Kalauni, P. Parashar, R. Guérout, G.-L. Ingold, A. Lambrecht and S. Reynaud, Fortschr. Phys. 65, 1600047 (2017).

37. G. Bimonte, T. Emig, M. Kardar and M. Krüger, Ann. Rev. Cond. Matt. Phys. 8, 119 (2017).

38. C. Henkel, Zeitschr. Naturforsch. A 72, 99 (2017).

39. J. R. Torgerson and S. K. Lamoreaux, Phys. Rev. E 70, 047102 (2004).

40. G. Bimonte, Phys. Rev. E 73, 048101 (2006).

41. J. R. Torgerson and S. K. Lamoreaux, Phys. Rev. E 73, 048102 (2006).

42. V. B. Svetovoy and R. Esquivel, J. Phys. A 39, 6777 (2006).

43. G. Bimonte, Phys. Rev. A 79, 042107 (2009).

44. P. R. Buenzli and P. A. Martin, Europhys. Lett. 72, 42 (2005).

45. B. Jancovici and L. Šamaj, Europhys. Lett. 72, 35 (2005).

46. X. L. Li, G. W. Ford and R. F. O'Connell, Phys. Rev. E 48, 1547 (1993).

47. F. S. S. Rosa, D. A. R. Dalvit and P. W. Milonni, Phys. Rev. A 81, 033812 (2010).

48. G.-L. Ingold, A. Lambrecht and S. Reynaud, Phys. Rev. E 80, 041113 (2009).

49. F. Haake and R. Reibold, Phys. Rev. A 32, 2462 (1985).

50. F. Intravaia and C. Henkel, Phys. Rev. Lett. 103, 130405 (2009).

51. C. Henkel and F. Intravaia, Int. J. Mod. Phys. A 25, 2328 (2010).

52. M. Bordag, Eur. Phys. J. C 71, 1 (2011). 
53. M. Bordag, Phys. Rev. D 85, 025005 (2012).

54. V. B. Svetovoy and M. V. Lokhanin, Phys. Rev. A 67, 022113 (2003).

55. V. B. Bezerra, G. L. Klimchitskaya, V. M. Mostepanenko and C. Romero, Phys. Rev. A 69, 022119 (2004).

56. S. A. Ellingsen, Phys. Rev. E 78, 021120 (2008).

57. R. S. Decca, E. Fischbach, G. L. Klimchitskaya, D. E. Krause, D. López, and V. M. Mostepanenko, Phys. Rev. D 68, 116003 (2003).

58. R. S. Decca, D. Lopez, E. Fischbach, G. L. Klimchitskaya, D. E. Krause and V. M. Mostepanenko, Ann. Phys. (N.Y.) 318, 37 (2005).

59. R. S. Decca, D. López, E. Fischbach, G. L. Klimchitskaya, D. E. Krause and V. M. Mostepanenko, Phys. Rev. D 75, 077101 (2007).

60. G. Bimonte, D. Lopez and R. S. Decca, Phys. Rev. B 93, 184434 (2016).

61. C.-C. Chang, A. A. Banishev, R. Castillo-Garza, G. L. Klimchitskaya, V. M. Mostepanenko, and U. Mohideen, Phys. Rev. B 85, 165443 (2012).

62. A. A. Banishev, G. L. Klimchitskaya, V. M. Mostepanenko, and U. Mohideen, Phys. Rev. Lett. 110, 137401 (2013).

63. Mingyue Liu, J. Xu, G. L. Klimchitskaya, V. M. Mostepanenko, and U. Mohideen, Phys. Rev. B 100, 081406(R) (2019). 